\title{
Hydrogen: A Novel Treatment Strategy in Kidney Disease
}

\author{
Bo Wang ${ }^{a}$ Zhuoshu Li ${ }^{b, c}$ Longfei Mao ${ }^{d}$ Mingyi Zhao $^{b}$ Bingchang Yang ${ }^{e}$ \\ Xiaowu Tao ${ }^{a}$ Yuxiang Li $^{\mathrm{a}}$ Guangming Yin ${ }^{\mathrm{a}}$ \\ aDepartment of Urology, The Third Xiangya Hospital, Central South University, Changsha, China; ${ }^{b}$ Department of \\ Pediatrics, The Third Xiangya Hospital, Central South University, Changsha, China; 'Xiangya School of Medicine, \\ Central South University, Changsha, China; ${ }^{\mathrm{d} B i o i n f o r m a t i c s ~ C e n t e r, ~ C o l l e g e ~ o f ~ B i o l o g y, ~ H u n a n ~ U n i v e r s i t y, ~ C h a n g s h a, ~}$ \\ China; 'Department of Critical Care Medicine, Central South University, Changsha, China
}

\section{Keywords}

Hydrogen · Kidney disease · Anti-inflammatory ·

Antioxidant $\cdot$ Cell death

\begin{abstract}
Background: Hydrogen is a chemical substance that has yet to be widely used in medicine. However, recent evidence indicates that hydrogen has multi-faceted pharmacological effects such as antioxidant, anti-inflammatory, and antiapoptotic properties. An increased number of studies are being conducted on the application of hydrogen in various diseases, especially those affecting the renal system. Summary: Hydrogen can be inhaled, as a gas or liquid, and can be administered orally, intravenously, or locally. Hydrogen can rapidly enter suborganelles such as mitochondria and nucleus by simple diffusion, producing reactive oxygen species (ROS) and triggering DNA damage. Hydrogen can selectively scavenge hydroxyl radical $(\cdot \mathrm{OH})$ and peroxynitrite $\left(\mathrm{ONOO}^{-}\right)$, but not other reactive oxygen radicals with physiological functions, such as peroxyanion $\left(\mathrm{O}_{2}^{-}\right)$and hydrogen peroxide $\left(\mathrm{H}_{2} \mathrm{O}_{2}\right)$. Although the regulatory effect of hydrogen on the signal transduction pathway has been confirmed, the specific mechanism of its influence on signal molecules re-
\end{abstract}

karger@karger.com www.karger.com/kdd

Karger $\stackrel{\text { ' }}{=}$
(C) 2022 The Author(s)

Published by S. Karger AG, Basel

This is an Open Access article licensed under the Creative Commons Attribution-NonCommercial-4.0 International License (CC BY-NC) (http://www.karger.com/Services/OpenAccessLicense), applicable to the online version of the article only. Usage and distribution for commercial purposes requires written permission. mains unknown. Although many studies have investigated the therapeutic and preventive effects of $\mathrm{H}_{2}$ in cellular and animal experiments, clinical trials are few and still far behind. As a result, more clinical trials are required to investigate the role of hydrogen in kidney disease, as well as the effect of its dose, timing, and form on the overall efficacy. Large-scale randomized controlled clinical trials will be required before hydrogen can be used to treat renal illnesses. Key Messages: This article reviews the mechanisms of hydrogen in the treatment of renal disease and explores the possibilities of its use in clinical practice.

(c) 2022 The Author(s).

Published by S. Karger AG, Basel

\section{Introduction}

Kidney disease is one of the most common diseases in humans. Common types of kidney disease include acute kidney injury (AKI), renal fibrosis, polycystic kidney disease, and renal cell carcinoma. There are many treatment options available for kidney diseases, including drug therapy, kidney dialysis, and kidney transplantation. In recent years, increasing attention has been paid to research using hydrogen to treat these diseases. Hydrogen exhibits 
Fig. 1. Biological effects of $\mathrm{H}_{2} . \mathrm{H} 2$ exhibits selective anti-oxidative, anti-inflammatory activities, and plays a role in the regulation of mitochondria, immune system, and ER stress. $\mathrm{H} 2$ selectively scavenges $\bullet \mathrm{OH}$ and $\mathrm{ONOO}^{-}$, upregulates Nrf2/ARE, SOD, CAT, GSH, and HO-1, and downregulates the expression of pro-inflammatory and inflammatory cytokines that include TNF- $\alpha$, NF-k $\beta$, IL- $1 \beta$, IL-6, IL-8, and IL10 , as well as downregulates ER stress-related factors that include GRP78 and TRAF2. H2 also regulates ROS to help maintain the function of mitochondria. Finally, $\mathrm{H} 2$ reduces the secretion of cytokines and vasoactive factors and maintains the balance of the immune system.

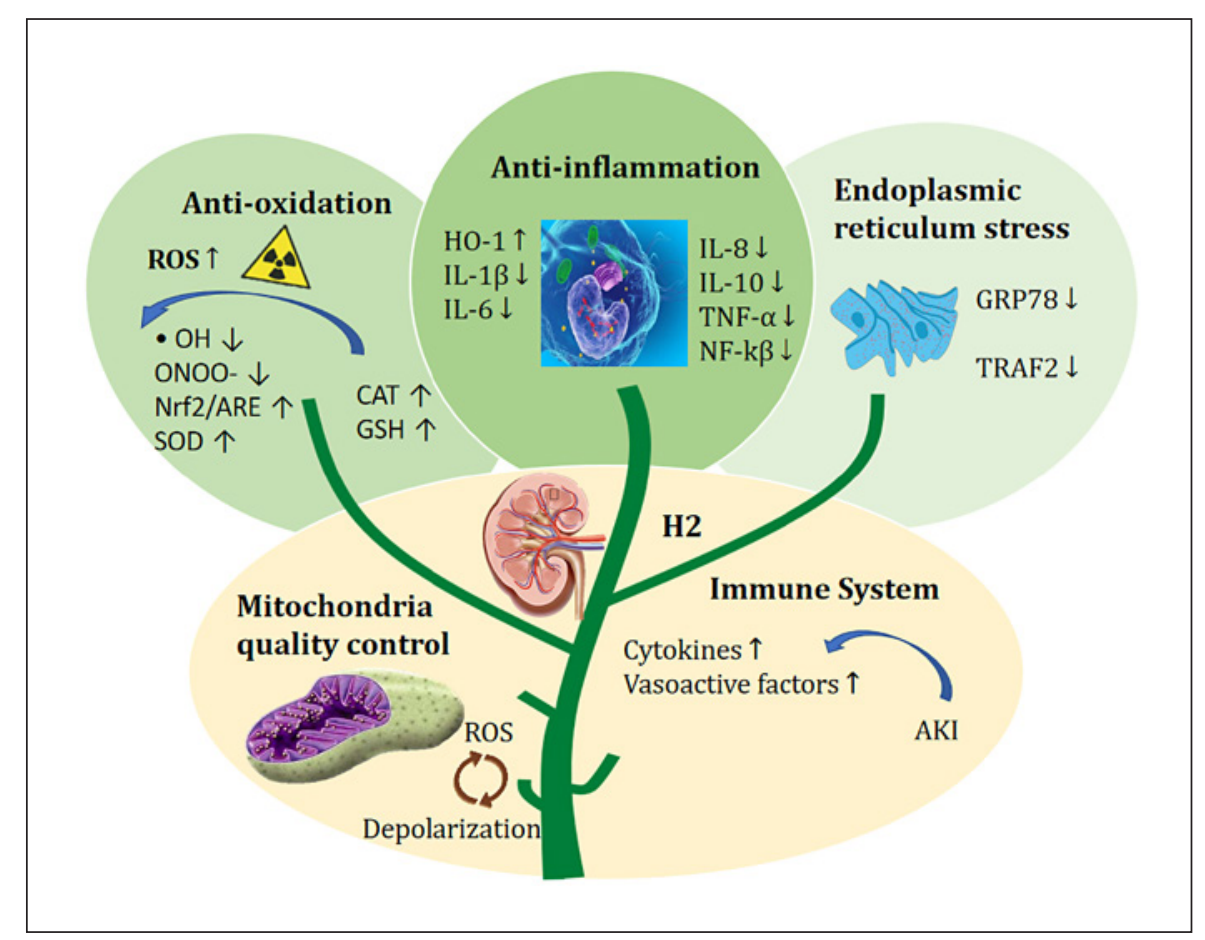

strong reducing properties and is frequently used as a reducing agent in chemical reactions.

Therapeutic applications of $\mathrm{H}_{2}$ were first reported in 1975 , when tumors' size reduced significantly when hairless albino mice with squamous cell carcinoma were exposed to a mixture of $2.5 \%$ oxygen and $97.5 \%$ hydrogen at a total pressure of 8 atmospheres for up to 2 weeks [1]. In 2007, Ohsawa et al. [2] proposed for the first time that hydrogen gas had antioxidant and antiapoptotic properties that could protect the brain against ischemia-reperfusion (I/R) injuries (IRIs) and stroke by selectively neutralizing hydroxyl radicals. Hydrogen has attracted much attention due to its potential therapeutic effect on kidney diseases. Many clinical and experimental models of kidney disease, as well as accumulating evidence in other biomedical domains, have demonstrated that hydrogen can act as a scavenging agent to selectively alleviate reactive oxygen species (ROS). Inhalation of hydrogen gas or use of hydrogen-containing aqueous solution can exert a powerful cellular protective effect.

In this review, we will focus on the physiological roles of hydrogen in kidney diseases, its potential as a therapeutic strategy, and the mechanisms that may be implicated in its protective effects. In addition, the findings of recent studies on hydrogen in various kidney disease models are discussed.

Hydrogen and Kidney Disease

\section{Action Mechanisms}

To fully explain the preventive and therapeutic effects of $\mathrm{H}_{2}$, Figures 1 and 2 show the biological effects of $\mathrm{H}_{2}$ and its impact on cell death.

\section{Biological Effects of $\mathrm{H}_{2}$}

Anti-Oxidation

$\mathrm{H}_{2}$, a potent oxidant, can react with nucleic acids without discrimination. The process of decomposing substances and releasing energy within or outside the body is known as oxidation. The intense oxidation of intracellular ROS or free radicals causes oxidative stress (OS), a state of imbalance between oxidation and antioxidant effects in the body which is considered to be a major contributor to aging and disease onset and progression. Acute OS induced by I/R or inflammation causes serious damage to tissues, and chronic OS is recognized as a causal agent of many kidney diseases. Hydrogen can scavenge free radicals. Hydrogen selectively reduces hydroxyl radicals $(\bullet \mathrm{OH})$ and peroxynitrite $\left(\mathrm{ONOO}^{-}\right)$, which are strong oxidants that react indiscriminately with nucleic acids, lipids, and proteins, resulting in DNA fragmentation, lipid peroxidation, and protein inactivation [2]. The potential therapeutic benefits of $\mathrm{H}_{2}$ were first described in 2007 . Ohsawa et al. [2] found that $\mathrm{H}_{2}$ selectively reduced $\bullet \mathrm{OH}$ and $\mathrm{ONOO}^{-}$in cultured cells and examined whether $\mathrm{H}_{2}$ 
Fig. 2. Effects of $\mathrm{H}_{2}$ on cell death. Activities of $\mathrm{H} 2$ include inhibition of apoptosis, the regulation of autophagy, ferroptosis, and pyroptosis; however, the specific mechanism is still unknown.

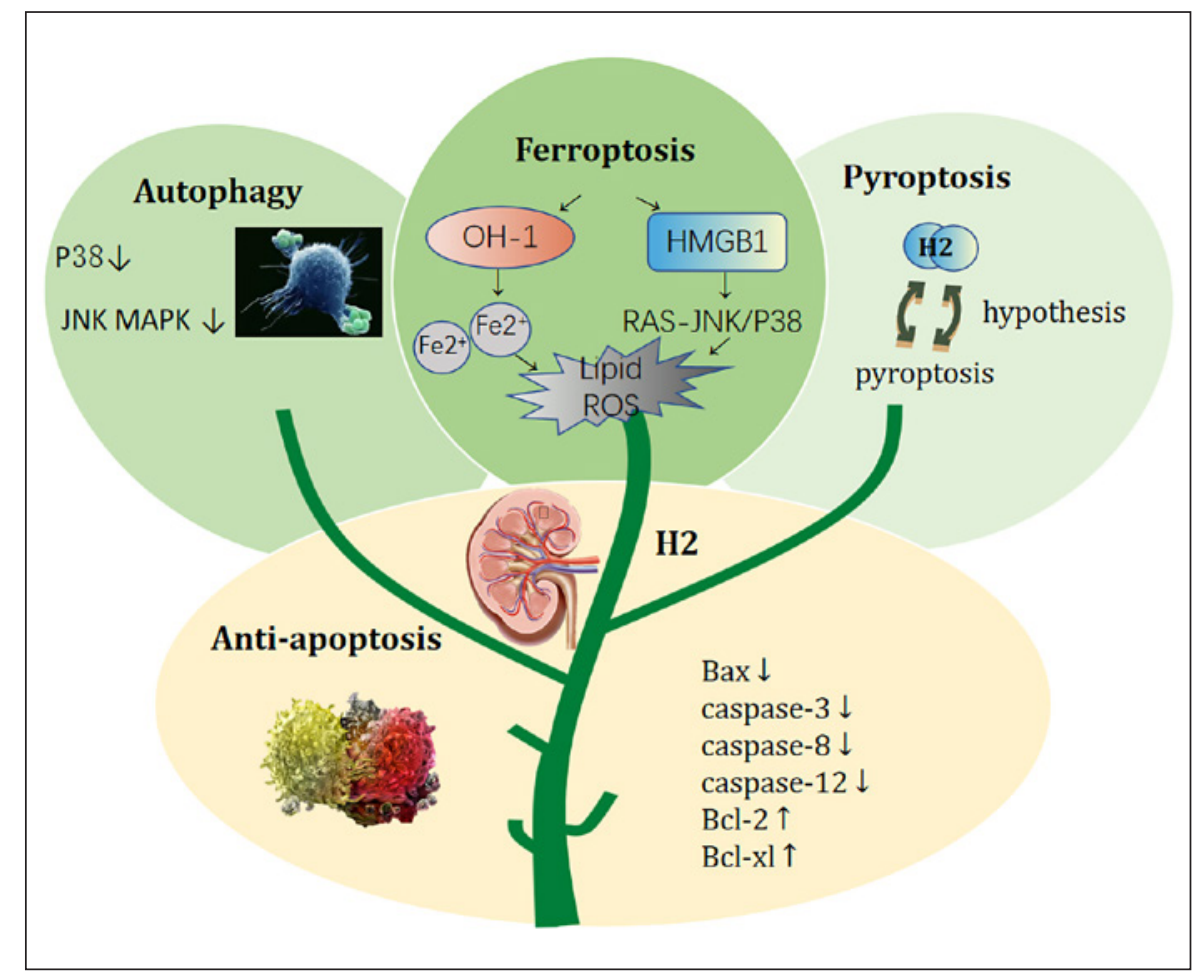

reduced the oxidized forms of biomolecules involved in metabolic oxidation-reduction reactions in cell-free assays. At room temperature and neutral $\mathrm{pH}$, solutions saturated with $\mathrm{H}_{2}$ did not reduce the oxidized forms of nicotinamide adenine dinucleotide, the oxidized form of flavin adenine dinucleotide, or the oxidized form of cytochrome $\mathrm{C}$. Thus, $\mathrm{H}_{2}$ had no effect on oxidation-reduction metabolism or the levels of $\mathrm{O}_{2}^{-}, \mathrm{H}_{2} \mathrm{O}_{2}$, and $\mathrm{NO}$, all of which play important physiological roles at low concentrations. As regulatory signaling molecules, they participate in many signal transduction cascades and regulate biological processes such as apoptosis, cell proliferation, and differentiation. Thus, $\mathrm{H}_{2}$ has selectiveantioxidant properties that protect the brain against I/R injury by specifically neutralizing $\cdot \mathrm{OH}$ and $\mathrm{ONOO}^{-}$but not $\mathrm{O}_{2}^{-}$, $\mathrm{H}_{2} \mathrm{O}_{2}$, and $\mathrm{NO}$ [2]. Unlike many strong reducing antioxidants, it does not affect essential physiological processes of the body [3]. In addition to directly neutralize free radicals, hydrogen can activate the Nrf2/ARE pathway in vivo and in vitro, increasing the transcription of downstream antioxidant enzyme genes, such as heme oxygenase 1 (HO-1), and contributing to the antioxidant activity [4]. Hydrogen can also increase the expression of endogenous superoxide dismutase, catalase [5], and reduced glutathione (GSH) [6], all of which help to reduce oxidative damage. Hydrogen plays various roles in the selective scavenging of ROS, downstream cascade signal transduction, and regulation of antioxidant enzyme activity. Therefore, studies on the antioxidant mechanisms of hydrogen and its influence in reducing damage due to OS in the kidneys provide a new direction for the future treatment of kidney diseases.

\section{Immune System}

Basic and clinical studies have shown that $\mathrm{H}_{2}$ is an important regulator with antioxidant, anti-inflammatory, and antiapoptotic effects [7]. $\mathrm{H}_{2}$ showed to have an antiinflammatory effect in LPS-activated macrophages, according to Hong et al. [8], by inhibiting the release of proinflammatory cytokines and increasing the release of the anti-inflammatory cytokine, which could be mediated by HO-1. Subsequently, some researchers found that resuscitation with hydrogen-enriched saline could significantly improve tissue and organ damage, potentially lowering the inflammatory responses and OS by inhibiting the expression and activation of NF- $\kappa B$ [9]. $\mathrm{H}_{2}$ was also able to inhibit the expression of pro-inflammatory cytokines during inflammation and reduce the early overexpression of pro-inflammatory cytokines, such as interleukin (IL)$1 \beta$, IL-6, IL-8, IL-10, and tumor necrosis factor-alpha (TNF- $\alpha$ ) in many animal models [10]. The network of inflammatory mediators and inflammatory effector cells 
and the imbalance between pro-inflammatory cytokines and anti-inflammatory cytokines play an important role in the occurrence and progression of kidney diseases. Thus, the treatment of inflammatory kidney diseases from the perspective of pro-inflammatory cytokines and anti-inflammatory cytokines may be an interesting route for further investigations of the role of hydrogen in regulating and maintaining homeostasis in inflammatory kidney disease.

Diabetic nephropathy (DN) is a serious complication of diabetes mellitus. Metabolic disorders can occur in DN and manifest as local inflammation of the kidney that can lead to fibrosis and structural remodeling of the organ. Therefore, tackling the immune-mediated inflammation is very significant for the treatment of DN [11]. In AKIs, such as renal artery infarction or toxin-mediated kidney injury, immune cells in the kidney are activated. Damaged renal epithelial cells activate the stress response pathways, leading to the secretion of cytokines and vasoactive factors, resulting in immunopathological damage [12]. Hydrogen, on the other hand, can suppress the production of immunoreactive substances [13]. We speculate that hydrogen has a future in immunological kidney disease as it is closely linked to significant antioxidant effects. However, there is currently no clinical data to confirm this. Further studies are required to confirm this theory.

\section{Regulation of ER Stress}

Endoplasmic reticulum (ER) stress occurs when pathological stress induces an accumulation of unfolded proteins in the ER. Zhao et al. [14] observed that the inhaled hydrogen significantly reduced the ER stress-related protein levels and alleviated tissue damage in myocardial IRI. Later, it was found that the mixture of $\mathrm{H}_{2}$ and $\mathrm{O}_{2}$ could inhibit ER stress via the PKR-like ER-localized eIF2 $\alpha$ kinase-eukaryotic initiation factor 2 alpha-activating transcription factor 4 (PERK-eIF2a-ATF 4), inositol-requiring enzyme 1-X-box binding protein 1 (IRE 1-XBP1), and ATF 6 pathways. A study on the relationship between $\mathrm{H}_{2}$ and ER stress in rats with IRI found that $\mathrm{H}_{2}$ reduced the expression of GRP78 and TNF receptor-associated factor 2 [15], indicating that the protective effects of $\mathrm{H}_{2}$ on myocardial IRI are related to a decrease in ER stress. In terms of kidney disease, studies focusing on the influence of hydrogen as a down-regulator of ER stress are scarce; however, we consider this to be an interesting future line of research.

\section{Mitochondria Quality Control}

The urate-induced inflammasome pathway in urate nephropathy involves the entry of urate crystals into in- tracellular lysosomes, which are decomposed to produce mitochondrial ROS, activating NLRP3 inflammasomes [16]. Excessive ROS production is due to the release of calcium in ER, leading to mitochondrial depolarization, and mitochondrial membrane potential loss. Mitochondrial depolarization leads to the release of more ROS in the mitochondria. The negative regulation of ROS by hydrogen may help to maintain the mitochondrial function $[13,17]$. Currently, the regulation of the mitochondrial function using hydrogen has not been yet reported in the treatment of kidney diseases, but this could be a promising research area.

\section{Effects of $\mathrm{H}_{2}$ on Cell Death}

Anti-apoptosis

Apoptosis is a form of programmed cell death characterized by cell shrinkage, apoptotic body formation, karyorrhexis, and chromatin condensation. Apoptosis can be induced by both endogenous and exogenous pathways. $\mathrm{H}_{2}$ plays an antiapoptotic role by upregulating or downregulating apoptotic-related factors. $\mathrm{H}_{2}$ also inhibits the expression of the proapoptotic factors B-cell lymphoma2 -associated X-protein (Bax), caspase- $3,-8$, and -12, and upregulates the antiapoptotic factors B-cell lymphoma-2 (Bcl-2) and B-cell lymphoma-extra-large (Bcl-xl) [18]. It was found that the intraperitoneal injection of a hydrogen-rich solution $10 \mathrm{~min}$ before skeletal muscle in I/R could reduce the expression of apoptotic protein Bax and cytochrome $\mathrm{C}$ (which can activate the expression of caspase-induced caspase cascade reaction), and increase the expression of antiapoptotic protein $\mathrm{Bcl}-2$, thus alleviating skeletal muscle injury after reperfusion [19]. Other scholars have found that an intraperitoneal injection of a hydrogen-rich solution can significantly increase the survival rate of skin flap, which is related to the regulation of ASK-1/JNK pathway and Bax/Bcl-2 ratio [20]. However, the exact mechanism of the antiapoptotic effect of hydrogen in kidney disease is not completely clear.

\section{Autophagy}

Autophagy is an important mechanism for maintaining cell homeostasis and promotes energy utilization [21]. Autophagy has a significant impact on renal function and homeostasis. In kidney studies using adult animals, autophagy has been found to affect different types of renal cells to help maintain kidney pathology and homeostasis [22]. Excessive autophagy or autophagy-related stress can aggravate the inflammatory damage of tissues and organs. When protein aggregates become toxic, autophagy is activated, and once excessive autophagy 
Table 1. The effect of $\mathrm{H} 2$ on kidney diseases

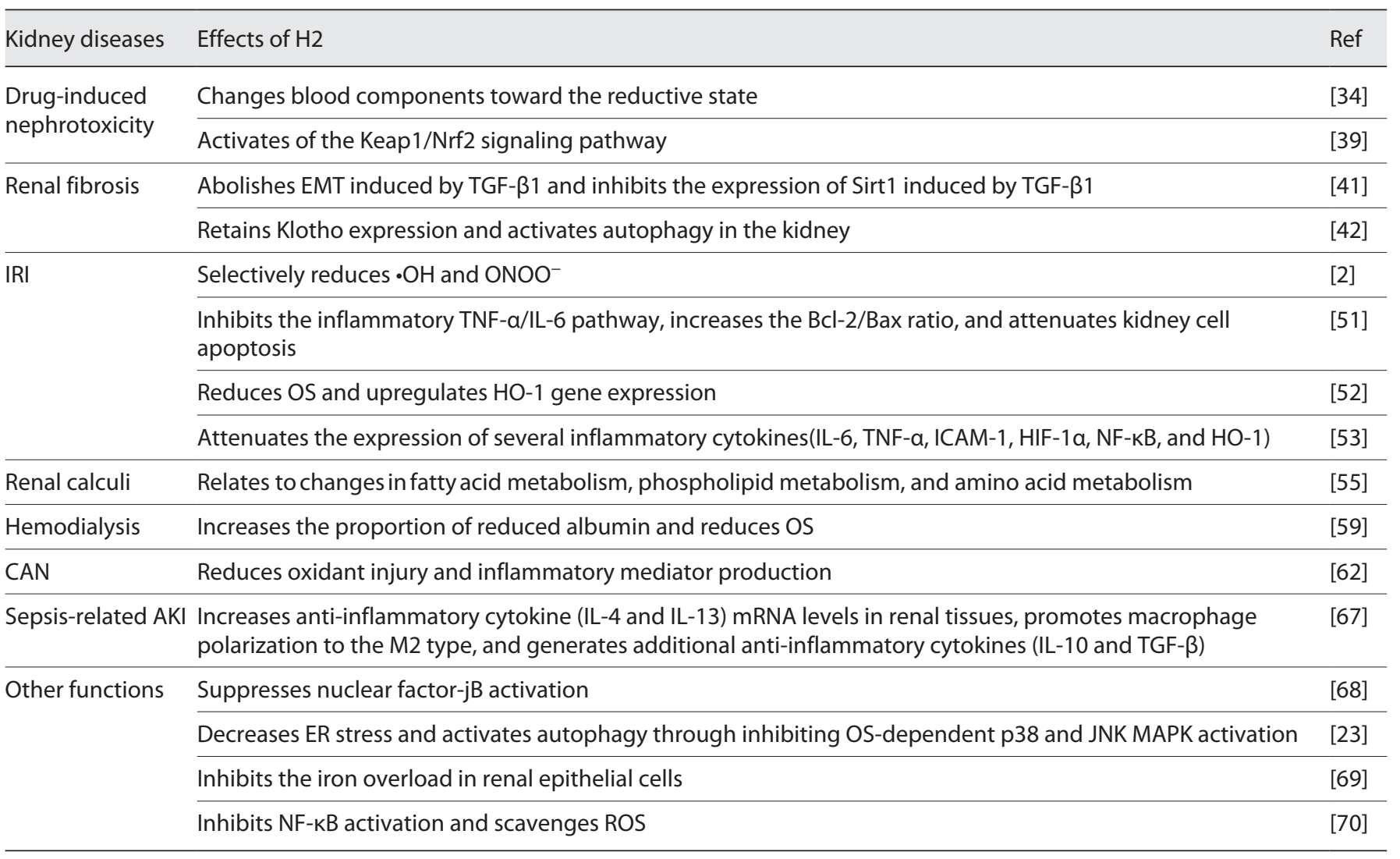

EMT, epithelial to mesenchymal transition.

causes tissue damage, autophagy is blocked. $\mathrm{H}_{2}$ appears to play a regulatory role [7]. Guan et al. [23] revealed that $\mathrm{H}_{2}$ was able to ameliorate chronic intermittent hypoxia (CIH)-induced kidney injury by decreasing ER stress and activating autophagy by inhibiting OS-dependent p38 and JNK MAPK activation. However, additional animal and clinical trials are needed, in order to further explore the underlying mechanisms.

\section{Pyroptosis}

The definition of pyroptosis was first proposed in 2012. It is accompanied by the accumulation of reactive lipid oxygen catalyzed by iron, cell apoptosis, necrosis, and autophagy [24]. In recent years, there has been evidence that pyroptosis plays an important role in the occurrence and development of AKI [25, 26], renal fibrosis [27], polycystic kidney disease [28], and renal cell carcinoma $[24,29]$. Since there is a considerable overlap between hydrogen regulation and pyroptosis pathways, we hypothesized that hydrogen could play a role in the treat- ment of renal diseases by regulating pyroptosis pathways. There is very little research on this matter, so additional research may be needed to confirm this hypothesis.

\section{Ferroptosis}

Ferroptosis is morphologically, biochemically, and genetically distinct from apoptosis, autophagy, and various forms of necrosis. It is characterized by the iron-dependent accumulation of ROS and lipid peroxidation, and it can be suppressed by iron chelators, lipophilic antioxidants, and inhibitors of lipid peroxidation [24]. A recent study [30] showed that HMGB1 is a novel ferroptosis regulator via the RAS-JNK/p38 pathway. Yu et al. [31] demonstrated that the treatment with $\mathrm{H}_{2}$ in the form of gas reduced the levels of HMGB1. Therefore, we speculate that $\mathrm{H}_{2}$ may be a potential drug agent for kidney diseases. Ferroptosis is an iron-dependent form of regulation of nonapoptotic cell death, which contributes to damage in models of AKI. HO-1, a potential source of intracellular iron, is a cytoprotective enzyme induced by cellular stress. 
Due to its antiapoptotic and anti-inflammatory properties [32], it has a protective effect on AKI. HO-1 activity can be increased by hydrogen. A recent study [32] demonstrated that HO-1-deficient renal epithelial cells were more sensitive to ferroptosis, indicating that free iron produced by HO-1 does not promote ferroptosis itself, and $\mathrm{HO}-1$ has an anti-ferroptotic effect. Although the mechanisms behind the hydrogen's effect on ferroptosis have yet to be fully clarified, it may add new directions to the search for kidney disease treatments.

\section{The Role of Kidney Diseases}

The effects of $\mathrm{H}_{2}$ in kidney diseases are shown in Table 1 .

\section{Drug-Induced Nephrotoxicity}

Drug-induced nephrotoxicity is becoming increasingly common today. A common cause of renal toxicity can be drugs, such as antibiotics or antitumor drugs. Cisplatin, a widely used cancer chemotherapy agent, is effective against many solid tumors, including lung, ovary, and breast cancer. Nephrotoxicity is the main dose-limiting toxicity of cisplatin. Despite saline hydration and diuresis, the incidence of nephrotoxicity is in the range of $20-30 \%$ [33]. Cisplatin has a high affinity for $\mathrm{SH}$ (sulph-hydryl) groups, in addition to DNA, its the primary target [34]. The interaction between cisplatin and $\mathrm{SH}$ groups leads to GSH depletion, resulting in the reduction of the cellular antioxidant system and accumulation of ROS and its products [34]. Cisplatin is mainly accumulated in the kidney as it is excreted mostly through this organ [35]. The accumulation of cisplatin and the generation of ROS in the kidney may be attributed to cisplatin-induced nephrotoxicity. Nakashima-Kamimura et al. [36] showed that the inhalation of hydrogen gas has a protective effect against cisplatin. For acute and strong OS induced by $\mathrm{I} / \mathrm{R}, 1 \%$ of hydrogen gas provides sufficient protection, while the inhalation of $1 \%$ or $2 \%$ hydrogen gas may be applicable for short-term treatments. In addition to hydrogen gas, this study demonstrated that drinking hydrogen water ad libitum was also effective in obtaining a significant protective effect. Subsequently, some researchers confirmed that hydrogenrich water (HW) ameliorates renal dysfunction caused by cisplatin-induced nephrotoxicity in rats, using dynamic contrast-enhanced CT and blood oxygenation level-dependent magnetic resonance imaging [37, 38]. Also, HW restored the redox equilibrium, suppressed OS damage, and improved kidney function induced by cyclosporine A via activation of the Keap1/Nrf2 signaling pathway [39]. The findings above [36-39] confirm that HW can be a promising therapeutic strategy for treating renal diseases and preventing clinical chronic allograft nephrotoxicity following organ transplantation.

\section{Renal Fibrosis}

Renal fibrosis, characterized by the accumulation of scars within the parenchyma, represents a common final pathway of chronic and progressive nephropathy. Renal fibrosis affects half of adults over the age of 70 and $10 \%$ of the world's population [40]. Therefore, actively exploring the pathogenesis and defense measures of renal fibrosis is important to improve the prognosis of kidney disease. Xing et al. [41] used HW to treat a Balb/C mouse model of renal fibrosis constructed by unilateral ureteral obstruction. Their study showed that HW alleviated unilateral ureteral obstruction-induced renal fibrosis. In the cell model, HW prevented the HK-2 cells from transforming to epithelial to mesenchymal transition induced by TGF- $\beta 1$, in which Sirt 1 other than TGF- $\beta 1 /$ Smad 2 pathway was involved. The study implies a therapeutic potential of hydrogen against fibrosis, with Sirt1 being a latent target for treatment and diagnosis of fibrosis. Chen et al. [42] found that hydrogen-rich saline (HRS) showed a protective effect in the prevention of renal injury and could inhibit renal fibrosis after an IR injury in mice. HRS might be exerted via retaining Klotho expression and activating autophagy in the kidney. Future research should aim to find out a non-invasive method for treating renal fibrosis.

\section{Ischemia-Reperfusion Injury}

IRI, which is unavoidable in organ transplantation, is severely detrimental to renal graft function and survival. One of the major events in ischemia-reperfusion is the generation of cytotoxic oxygen radicals, which lead to cellular injury by inducing DNA damage, protein oxidation, lipid peroxidation, and apoptosis [43]. In 2001, Noiri et al. [44] demonstrated that the generation of ROS and NO in IRI can result in the formation of a cytotoxic metabolite, peroxynitrite $\left(\mathrm{ONOO}^{-}\right)$, which can cause lipid peroxidation and DNA damage. A rapid transition from ischemic condition to reperfusion causes OS damage [45]. To mimic ischemia, Ohsawa et al. [2] subjected neocortical cells to oxygen-glucose deprivation (OGD) under nitrogen or hydrogen gas for $60 \mathrm{~min}$, followed by reperfusion with medium containing $\mathrm{O}_{2}$ and glucose. HPF fluorescence showed that, 10 min after the comple- 
tion of OGD followed by reperfusion, $\bullet \mathrm{OH}$ levels notably increased in the absence of $\mathrm{H}_{2}$ but diminished when $\mathrm{H}_{2}$ was present. At $24 \mathrm{~h}$ after OGD and reperfusion, $\mathrm{H}_{2}$ increased neuron survival and vitality, indicating that $\mathrm{H}_{2}$ protected neurons against OS-induced cell death. In addition, 8-OHdG and malondialdehyde are main forms of DNA and lipid damage-induced ROS, respectively [43]. Given that $\mathrm{H}_{2}$ molecules can mitigate IRI by selectively removing ROS that lead to oxidative damage to DNA, lipids, and proteins, Kawamura et al. [43] evaluated OS by monitoring urinary $8-\mathrm{OHdG}$ and serum malondialdehyde levels. 8-OHdG and malondialdehyde, which are peroxidation products of DNA and lipids, respectively, are widely used as OS markers [46-48]. The treatment with nano-Si led to a reduction in the increased urinary 8-OHdG and malondialdehyde levels due to IRI. To sum up, $\bullet \mathrm{OH}$ and $\mathrm{ONOO}^{-}$are the major ROS that contributes to IRI, while 8-OHdG and malondialdehyde can be used as main OS markers to IRI. Since the discovery of the selective antioxidant properties of molecular hydrogen $\left(\mathrm{H}_{2}\right)$ in 2007 , several studies have shown that hydrogen has beneficial effects in a variety of animal models of OS in vivo $[2,49,50] . \mathrm{H}_{2}$ treatment has been shown to abrogate IRI following warm and cold ischemia, and thus, it has been identified as a potential therapy to improve kidney transplantation outcomes [8]. Li et al. [51] induced a rat model of renal IRI and found that the treatment with HRS solution (HRSS) significantly reduced interstitial congestion, edema, and hemorrhage compared with saline treatment. HRSS could promote renal function recovery after IRI in rats by inhibiting the inflammatory TNF- $\alpha$ /IL- 6 pathway, increasing the Bcl$2 /$ Bax ratio, and attenuating kidney cell apoptosis. Another study [52] reported that HRS improved the renal response to I/R in aged rats, possibly by reducing OS and upregulating HO-1 gene expression. In addition, Nishida et al. [53] conducted a study to evaluate the effects of a mixture of $\mathrm{H}_{2}$ gas and $\mathrm{CO}$ gas (dual gas) in comparison with hydrogen gas (H2: 2\%) alone on I/R renal injury. They observed the cytoprotective effects of dual treatment in comparison with $\mathrm{H}_{2}$ treatment and $\mathrm{I} / \mathrm{R}$ renal injury in terms of superoxide radical scavenging activity and histochemical features. Rats given dual treatment showed significant reduction in both blood urea nitrogen and the expression of several inflammatory cytokines (IL-6, TNF- $\alpha$, ICAM-1, HIF- $1 \alpha$, NF- $\kappa$ B, and HO1). Although the mechanism of the protective effect of hydrogen remains unclear, the application of hydrogen therapy may be a simple, safe, economical, and effective new method for protecting against kidney injury.

\section{Renal Calculi}

Kidney stones, caused by the abnormal accumulation of crystalline substances (calcium oxalate - $\mathrm{CaOx}$ - being the most common), are one of the most common diseases of the urinary system. In order to evaluate the protective effect and underlying mechanism of hydrogen gas $\left(\mathrm{H}_{2}\right)$ in glyoxylate-induced renal $\mathrm{CaOx}$ crystal deposition in mice, Peng et al. [54] established a rodent renal $\mathrm{CaOx}$ crystal deposition model by an intra-abdominal injection of glyoxylate (precursor of oxalate) for 5 days. According to the results of the levels of urine calcium excretion, renal calcium deposition, serum excretion of kidney injury molecule-1 (KIM-1) assay, and TUNEL assay, the inhalation of $\mathrm{H}_{2}$ successfully decreased the crystallization of $\mathrm{CaOx}$ and protected against renal injury. Crystal deposition in the kidneys is associated with OS. OS leads to apoptosis and necrosis of renal tubular epithelial cells. This is a key element for the renal tubular epithelial cell damage [55] and contributes to the renal crystal deposition and occurrence of renal stones [56], due to the production of ROS [57]. ROS are considered to be cytotoxic and can damage a variety of macromolecules [58]. Serum metabolomics studies in mice [55] showed that $\mathrm{H}_{2}$ protects against renal injury caused by crystallization of $\mathrm{CaOx}$, which may be related to changes in the fatty acid metabolism, phospholipid metabolism, and amino acid metabolism. More research, however, is needed to gain a better understanding of the molecular mechanisms of $\mathrm{CaOx}$ induced renal injury and the therapeutic effects of hydrogen gas.

\section{Hemodialysis}

Increased OS is associated with severe cardiovascular disease and premature death in patients treated with hemodialysis (HD). During HD, the exposure of the dialysis membrane to blood can enhance the production of OS [59]. In a clinical study [59], 8 patients with regular hemodialysis were alternately treated with standard dialysate and dialysate dissolved with high concentration of hydrogen, and the results showed that dialysate containing hydrogen could reduce the level of OS in hemodialysis patients, which may reduce the incidence of cardiovascular diseases. Thus, dialysate containing hydrogen can provide a new treatment for the control of uremia. In another study [60], Nakayama et al. [60] developed a novel hemodialysis (E-HD) system delivering an $\mathrm{H}_{2}$ (30-80 $\mathrm{ppb}$ )-enriched dialysis solution by water electrolysis and conducted a nonrandomized, nonblinded, prospective observational study exploring its clinical impact. It was concluded that E-HD was an independent significant fac- 
tor in reducing the risk of the primary events of all-cause mortality and development of nonlethal cardio-cerebrovascular events. Thus, the application of $\mathrm{H}_{2}$-enriched solutions may ameliorate $\mathrm{OS}$ during $\mathrm{HD}$, although more research is needed to determine its clinical impact.

\section{Chronic Allograft Nephropathy}

Chronic allograft nephropathy (CAN), also called interstitial fibrosis and tubular atrophy of unknown etiology (IF/TA), remains one of the most vexing clinical entities for the renal transplant physicians to treat, as it is resistant to current treatment modalities and is a major cause of long-term graft loss [61]. Therefore, potential new approaches to the treatment of CAN such as the one described by Cardinal et al. [62] are of great interest. The addition of hydrogen to drinking water reduced the severity of CAN and increased graft survival, indicating that the mechanism may be due to a reduction in ROS. Therefore, oral HW is an effective antioxidant and anti-inflammatory agent that prevented CAN, improved the survival rate of rat allograft kidney transplantation, and may have therapeutic value in transplantation.

\section{Sepsis-Related AKI}

AKI is common in the early stages of sepsis, with an incidence of 55-73\% [62], independently associated with mortality [63]. Approximately $70 \%$ of septic AKI cases are fatal. Thus, kidney protection is critical for these patients' survival [64]. Excessive inflammatory response and OS are considered to be the main mechanisms of septic AKI [65]. Recently, it has been discovered that reducing inflammation is critical in sepsis therapy, suggesting a new therapeutic concept for preventing septic AKI [66]. Yao et al. [67] explored the renal protective effects of aerosol inhalation of a hydrogen-rich solution in a mouse model of septic AKI. Septic AKI was induced by a 18-h cecal ligation and puncture. AKI occurred during the early stage of sepsis and was characterized by increased blood urea nitrogen and serum creatinine levels, pathological changes, renal fibrosis and renal tubular epithelial cell apoptosis, macrophage infiltration, and M1 macrophage-associated pro-inflammatory cytokine (IL6 and TNF- $\alpha$ ) generation in renal tissues. Aerosol inhalation of the HRS increased anti-inflammatory cytokine (IL-4 and IL-13) mRNA levels in renal tissues and promoted macrophage polarization to the M2 type, which generated additional anti-inflammatory cytokines (IL10 and TGF- $\beta$ ). Ultimately, aerosol inhalation of HRS protected the kidneys and increased survival among septic mice. Thus, HRS aerosol inhalation appears to be highly beneficial for renal protection and inflammation reduction in septic AKI.

\section{Other Functions}

Xin et al. [68] investigated the protective effect of HW on renal injury in spontaneously hypertensive rats (SHR). The 8-week-old male SHR and age-matched Wistar-Kyoto rats were randomized into HW-treated and vehicletreated group. Although HW had no significant effect on blood pressure, it significantly ameliorated renal injury, reducing the formation of ROS, suppressing NADPH oxidase activity, and upregulating the activities of superoxide GSH peroxidase, dismutase, GSH-S-epoxide transferase, and catalase. Treatment with HW in SHR-depressed expression of pro-inflammatory cytokines such as IL-6, IL-1b, TNF-a, and macrophage chemoattractant protein 1 , which may be mediated by inhibiting the activation of the nuclear factor- $\kappa \mathrm{B}$. Furthermore, HW treatment had a protective effect on mitochondrial function including adenosine triphosphate formation and membrane integrity in SHR. To sum up, HW is a promising strategy for reducing renal injury when used in conjunction with anti-hypertensive therapy.

Guan et al. [23] reveal that $\mathrm{H}_{2}$ can ameliorate $\mathrm{CIH}$ induced kidney injury by decreasing ER stress and activating autophagy through inhibiting OS-dependent $\mathrm{p} 38$ and JNK MAPK activation. Increased expression of renal transferrin receptor and divalent metal transporter-1 expression, as well as reduced ceruloplasmin expression may be possible causes for iron accumulation in the proximal tubule epithelial cells of rats after $\mathrm{CIH}$ treated. Inhalation of $\mathrm{H}_{2}$ could prevent renal injury induced by $\mathrm{CIH}$ via inhibition of iron overload in renal epithelial cells, which is involved in the mechanism of OS-mediated injury [69].

In addition, Shi et al. [70] demonstrated that HRS could attenuate acute renal injury in a sodium taurocholate-induced severe acute pancreatitis rat model. By inhibiting NF- $\kappa \mathrm{B}$ activation and scavenging ROS, HRS prevented the development of inflammatory cascade and eased renal oxidative damage. Homma et al. [71] found that contrast-induced AKI in rats was carried on using an intravenous injection of a contrast medium, loversol. During the injection of these reagents, the rats inhaled $\mathrm{H}_{2}$ gas or control gas. The inhalation of $\mathrm{H}_{2}$ was effective in ameliorating the severity of contrast-induced AKI in rats by reducing renal cell apoptosis and OS. 


\section{Conclusion}

At present, there have been few reports of hydrogen causing adverse reactions in cells. Hydrogen has been studied for its mutagenicity, genotoxicity, and subchronic toxicity. Saitoh et al. [72] investigated the toxic and side effects of neutral-pH hydrogen-enriched electrolyzed water on rats $(20 \mathrm{~mL} / \mathrm{kg} /$ day, lasting for 28 days). No toxicity was related to drinking hydrogen-rich electrolytic water in terms of clinical symptoms, hematology, and histopathology. Nakao et al. [73] conducted an open-label pilot study of drinking $\mathrm{HW}$, which showed that drinking HW down-regulated aspartate aminotransferase and alanine aminotransferase while increasing gamma-GSH transferase and total bilirubin. However, the changes in these parameters were limited to one gender, and the mean values of these parameters were within the normal acceptable reference range for both male and female subjects. So far, no studies have shown that hydrogen has a clear adverse impact on the treatment of renal disease.

To sum up, hydrogen plays a unique role in anti-oxidation, anti-inflammation, anti-apoptosis, and cell death. Several animal studies have now confirmed the therapeutic effect of hydrogen in kidney disease, such as renal calculi, renal fibrosis, and drug-induced nephrotoxicity. The specific molecular mechanisms behind the therapeutic effect of hydrogen remain unknown. Future research should focus on the negative effects of hydrogen and its specific mechanisms of action in kidney disease. Largescale clinical trials will be needed to demonstrate the effectiveness and cost-efficiency of hydrogen as a therapy against kidney disease.

\section{Conflict of Interest Statement}

The authors have no conflicts of interest to declare.

\section{Funding Sources}

This study was supported by the Science and Technology Innovation Plan of Hunan Province [2020SK53602].

\section{Author Contributions}

Bo Wang and Zhuoshu Li collected the literature and drafted the initial manuscript. Longfei Mao polished the language. Mingyi Zhao drafted the initial manuscript. Bingchang Yang directed the writing of the article. Xiaowu Tao drawn the figures and table. Yuxiang Li revised the manuscript and edited the language. Guangming Yin was the lead investigator. All authors approved the final manuscript as submitted and are accountable for all aspects of the work.

\section{References}

1 Dole M, Wilson FR, Fife WP. Hyperbaric hydrogen therapy: a possible treatment for cancer. Science. 1975;190(4210):152-4.

2 Ohsawa I, Ishikawa M, Takahashi K, Watanabe M, Nishimaki K, Yamagata K, et al. Hydrogen acts as a therapeutic antioxidant by selectively reducing cytotoxic oxygen radicals. Nat Med. 2007;13(6):688-94.

3 Ohta S. Recent progress toward hydrogen medicine: potential of molecular hydrogen for preventive and therapeutic applications. Curr Pharm Des. 2011;17(22):2241-52.

4 Xie Q, Li XX, Zhang P, Li JC, Cheng Y, Feng $\mathrm{YL}$, et al. Hydrogen gas protects against serum and glucose deprivation-induced myocardial injury in $\mathrm{H} 9 \mathrm{c} 2$ cells through activation of the NF-E2-related factor 2/heme oxygenase 1 signaling pathway. Mol Med Rep. 2014;10(2): 1143-9.

5 Wang F, Yu G, Liu SY, Li JB, Wang JF, Bo LL, et al. Hydrogen-rich saline protects against renal ischemia/reperfusion injury in rats. J Surg Res. 2011;167(2):e339-44.

6 Qian L, Cao F, Cui J, Huang Y, Zhou X, Liu S, et al. Radioprotective effect of hydrogen in cultured cells and mice. Free Radic Res. 2010; 44(3):275-82.
7 Huang CS, Kawamura T, Toyoda Y, Nakao A. Recent advances in hydrogen research as a therapeutic medical gas. Free Radic Res. 2010; 44(9):971-82.

8 Chen HG, Xie KL, Han HZ, Wang WN, Liu DQ, Wang GL, et al. Heme oxygenase-1 mediates the anti-inflammatory effect of molecular hydrogen in LPS-stimulated RAW 264.7 macrophages. Int J Surg. 2013;11(10):1060-6.

9 Wang X, Yu P, Yang Y, Liu X, Jiang J, Liu D, et al. Hydrogen-rich saline resuscitation alleviates inflammation induced by severe burn with delayed resuscitation. Burns. 2015;41(2): 379-85.

10 Liu W, Shan LP, Dong XS, Liu XW, Ma T, Liu Z. Combined early fluid resuscitation and hydrogen inhalation attenuates lung and intestine injury. World J Gastroenterol. 2013; 19(4):492-502.

11 Zheng Z, Zheng F. Immune cells and inflammation in diabetic nephropathy. J Diabetes Res. 2016;2016:1841690.

12 Yatim KM, Lakkis FG. A brief journey through the immune system. Clin J Am Soc Nephrol. 2015;10(7):1274-81.
13 Yang M, Dong Y, He Q, Zhu P, Zhuang Q, Shen J, et al. Hydrogen: a novel option in human disease treatment. Oxid Med Cell Longev. 2020;2020:8384742.

14 Zhang Y, Liu Y, Zhang J. Saturated hydrogen saline attenuates endotoxin-induced lung dysfunction. J Surg Res. 2015;198(1):41-9.

15 Huang T, Wang W, Tu C, Yang Z, Bramwell D, Sun X. Hydrogen-rich saline attenuates ischemia-reperfusion injury in skeletal muscle. J Surg Res. 2015;194(2):471-80.

16 Liu YQ, Liu YF, Ma XM, Xiao YD, Wang YB, Zhang MZ, et al. Hydrogen-rich saline attenuates skin ischemia/reperfusion induced apoptosis via regulating $\mathrm{Bax} / \mathrm{Bcl}-2$ ratio and $\mathrm{ASK}$ 1/JNK pathway. J Plast Reconstr Aesthet Surg. 2015;68(7):e147-56.

17 Zhong H, Song R, Pang Q, Liu Y, Zhuang J, Chen Y, et al. Propofol inhibits parthanatos via ROS-ER-calcium-mitochondria signal pathway in vivo and vitro. Cell Death Dis. 2018;9(10):932.

18 Zhao YS, An JR, Yang S, Guan P, Yu FY, Li W, et al. Hydrogen and oxygen mixture to improve cardiac dysfunction and myocardial pathological changes induced by intermittent hypoxia in rats. Oxid Med Cell Longev. 2019; 2019:7415212. 
19 Gao Y, Yang H, Chi J, Xu Q, Zhao L, Yang W, et al. Hydrogen gas attenuates myocardial ischemia reperfusion injury independent of postconditioning in rats by attenuating endoplasmic reticulum stress-induced autophagy. Cell Physiol Biochem. 2017;43(4):1503-14.

20 Isaka Y, Takabatake Y, Takahashi A, Saitoh T, Yoshimori T. Hyperuricemia-induced inflammasome and kidney diseases. Nephrol Dial Transplant. 2016;31(6):890-6.

21 Parzych KR, Klionsky DJ. An overview of autophagy: morphology, mechanism, and regulation. Antioxid Redox Signal. 2014;20(3): 460-73.

22 He L, Livingston MJ, Dong Z. Autophagy in acute kidney injury and repair. Nephron Clin Pract. 2014;127(1-4):56-60.

23 Guan P, Sun ZM, Luo LF, Zhou J, Yang S, Zhao YS, et al. Hydrogen protects against chronic intermittent hypoxia induced renal dysfunction by promoting autophagy and alleviating apoptosis. Life Sci. 2019;225:46-54.

24 Dixon SJ, Lemberg KM, Lamprecht MR, Skouta R, Zaitsev EM, Gleason CE, et al. Ferroptosis: an iron-dependent form of nonapoptotic cell death. Cell. 2012;149(5):106072.

25 Friedmann Angeli JP, Schneider M, Proneth B, Tyurina YY, Tyurin VA, Hammond VJ, et al. Inactivation of the ferroptosis regulator Gpx4 triggers acute renal failure in mice. Nat Cell Biol. 2014;16(12):1180-91.

26 Müller T, Dewitz C, Schmitz J, Schröder AS, Bräsen JH, Stockwell BR, et al. Necroptosis and ferroptosis are alternative cell death pathways that operate in acute kidney failure. Cell Mol Life Sci. 2017;74(19):3631-45

27 Hou W, Xie Y, Song X, Sun X, Lotze MT, Zeh HJ 3rd, et al. Autophagy promotes ferroptosis by degradation of ferritin. Autophagy. 2016; 12(8):1425-8.

28 Schreiber R, Buchholz B, Kraus A, Schley G, Scholz J, Ousingsawat J, et al. Lipid peroxidation drives renal cyst growth in vitro through activation of TMEM16A. J Am Soc Nephrol. 2019;30(2):228-42.

29 Yang WS, SriRamaratnam R, Welsch ME, Shimada K, Skouta R, Viswanathan VS, et al. Regulation of ferroptotic cancer cell death by GPX4. Cell. 2014;156(1-2):317-31.

30 Ye F, Chai W, Xie M, Yang M, Yu Y, Cao L, et al. HMGB1 regulates erastin-induced ferroptosis via RAS-JNK/p38 signaling in HL-60/ NRAS(Q61L) cells. Am J Cancer Res. 2019; 9(4):730-9.

31 Yu Y, Yang Y, Yang M, Wang C, Xie K, Yu Y. Hydrogen gas reduces HMGB1 release in lung tissues of septic mice in an $\mathrm{Nrf} 2 / \mathrm{HO}$ 1-dependent pathway. Int Immunopharmacol. 2019;69:11-8.

32 Adedoyin O, Boddu R, Traylor A, Lever JM, Bolisetty S, George JF, et al. Heme oxygenase-1 mitigates ferroptosis in renal proximal tubule cells. Am J Physiol Renal Physiol. 2018; 314(5):F702-14.
33 Rana MA, Khan RA, Nasiruddin M, Khan AA. Amelioration of cisplatin-induced nephrotoxicity by ethanolic extract of Bauhinia purpurea: an in vivo study in rats. Saudi J Kidney Dis Transpl. 2016;27(1):41-8.

34 Kuhlmann MK, Burkhardt G, Köhler H. Insights into potential cellular mechanisms of cisplatin nephrotoxicity and their clinical application. Nephrol Dial Transplant. 1997; 12(12):2478-80.

35 Yao X, Panichpisal K, Kurtzman N, Nugent K. Cisplatin nephrotoxicity: a review. Am J Med Sci. 2007;334(2):115-24.

36 Nakashima-Kamimura N, Mori T, Ohsawa I, Asoh S, Ohta S. Molecular hydrogen alleviates nephrotoxicity induced by an anti-cancer drug cisplatin without compromising antitumor activity in mice. Cancer Chemother Pharmacol. 2009;64(4):753-61.

37 Kitamura A, Kobayashi S, Matsushita T, Fujinawa $\mathrm{H}$, Murase K. Experimental verification of protective effect of hydrogen-rich water against cisplatin-induced nephrotoxicity in rats using dynamic contrast-enhanced $\mathrm{CT}$. $\mathrm{Br}$ J Radiol. 2010;83(990):509-14.

38 Matsushita T, Kusakabe Y, Kitamura A, Okada S, Murase K. Investigation of protective effect of hydrogen-rich water against cisplatininduced nephrotoxicity in rats using blood oxygenation level-dependent magnetic resonance imaging. Jpn J Radiol. 2011;29(7):50312.

39 Lu Y, Li CF, Ping NN, Sun YY, Wang Z, Zhao GX, et al. Hydrogen-rich water alleviates cyclosporine A-induced nephrotoxicity via the Keap1/Nrf2 signaling pathway. J Biochem Mol Toxicol. 2020;34(5):e22467.

40 Humphreys BD. Mechanisms of renal fibrosis. Annu Rev Physiol. 2018;80:309-26.

41 Xing Z, Pan W, Zhang J, Xu X, Zhang X, He $X$, et al. Hydrogen rich water attenuates renal injury and fibrosis by regulation transforming growth factor- $\beta$ induced Sirt1. Biol Pharm Bull. 2017;40(5):610-5.

42 Chen J, Zhang H, Hu J, Gu Y, Shen Z, Xu L, et al. Hydrogen-rich saline alleviates kidney fibrosis following AKI and retains Klotho expression. Front Pharmacol. 2017;8:499.

43 Kawamura M, Imamura R, Kobayashi Y, Taniguchi A, Nakazawa S, Kato T, et al. Oral administration of Si-based agent attenuates oxidative stress and ischemia-reperfusion injury in a rat model: a novel hydrogen administration method. Front Med. 2020;7:95

44 Noiri E, Nakao A, Uchida K, Tsukahara $H$, Ohno M, Fujita T, et al. Oxidative and nitrosative stress in acute renal ischemia. Am J Physiol Renal Physiol. 2001;281(5):F948-57.

45 Halestrap AP. Calcium, mitochondria and reperfusion injury: a pore way to die. Biochem Soc Trans. 2006;34(Pt 2):232-7.

46 Kato S, Yoshimura K, Kimata T, Mine K, Uchiyama T, Kaneko K. Urinary 8-hydroxy-2'-deoxyguanosine: a biomarker for radiation-induced oxidative DNA damage in pediatric cardiac catheterization. J Pediatr. 2015;167(6):1369-74.e1.
47 Wu LL, Chiou CC, Chang PY, Wu JT. Urinary 8-OHdG: a marker of oxidative stress to DNA and a risk factor for cancer, atherosclerosis and diabetics. Clin Chim Acta. 2004;339(12):1-9.

48 Tsikas D. Assessment of lipid peroxidation by measuring malondialdehyde (MDA) and relatives in biological samples: analytical and biological challenges. Anal Biochem. 2017; 524:13-30.

49 Buchholz BM, Kaczorowski DJ, Sugimoto R, Yang R, Wang Y, Billiar TR, et al. Hydrogen inhalation ameliorates oxidative stress in transplantation induced intestinal graft injury. Am J Transplant. 2008;8(10):2015-24.

50 Abe T, Li XK, Yazawa K, Hatayama N, Xie L, Sato B, et al. Hydrogen-rich University of Wisconsin solution attenuates renal cold ischemia-reperfusion injury. Transplantation. 2012;94(1):14-21.

51 Li J, Hong Z, Liu H, Zhou J, Cui L, Yuan S, et al. Hydrogen-rich saline promotes the recovery of renal function after ischemia/reperfusion injury in rats via anti-apoptosis and antiinflammation. Front Pharmacol. 2016;7:106.

$52 \mathrm{Xu} \mathrm{X,} \mathrm{He} \mathrm{X,} \mathrm{Liu} \mathrm{J,} \mathrm{Qin} \mathrm{J,} \mathrm{Ye} \mathrm{J,} \mathrm{Fan} \mathrm{M.} \mathrm{Protec-}$ tive effects of hydrogen-rich saline against renal ischemia-reperfusion injury by increased expression of heme oxygenase- 1 in aged rats. Int J Clin Exp Pathol. 2019;12(4):1488-96.

53 Nishida T, Hayashi T, Inamoto T, Kato R, Ibuki N, Takahara K, et al. Dual gas treatment with hydrogen and carbon monoxide attenuates oxidative stress and protects from renal ischemia-reperfusion injury. Transplant Proc. 2018;50(1):250-8.

54 Peng Z, Chen W, Wang L, Ye Z, Gao S, Sun X, et al. Inhalation of hydrogen gas ameliorates glyoxylate-induced calcium oxalate deposition and renal oxidative stress in mice. Int $J$ Clin Exp Pathol. 2015;8(3):2680-9.

55 Lu H, Ding J, Liu W, Peng Z, Chen W, Sun X, et al. UPLC/MS-based metabolomics investigation of the protective effect of hydrogen gas inhalation on mice with calcium oxalate-induced renal injury. Biol Pharm Bull. 2018; 41(11):1652-8.

56 Khan SR. Renal tubular damage/dysfunction: key to the formation of kidney stones. Urol Res. 2006;34(2):86-91.

57 Khan SR. Reactive oxygen species as the molecular modulators of calcium oxalate kidney stone formation: evidence from clinical and experimental investigations. J Urol. 2013; 189(3):803-11.

58 Jabłońska-Trypuć A, Pankiewicz W, Czerpak R. Traumatic acid reduces oxidative stress and enhances collagen biosynthesis in cultured human skin fibroblasts. Lipids. 2016; 51(9):1021-35.

59 Terawaki H, Zhu WJ, Matsuyama Y, Terada T, Takahashi Y, Sakurai K, et al. Effect of a hydrogen (H2)-enriched solution on the albumin redox of hemodialysis patients. $\mathrm{He}$ modial Int. 2014;18(2):459-66. 
60 Nakayama M, Itami N, Suzuki H, Hamada H, Yamamoto R, Tsunoda K, et al. Novel haemodialysis (HD) treatment employing molecular hydrogen $(\mathrm{H}(2))$-enriched dialysis solution improves prognosis of chronic dialysis patients: a prospective observational study. Sci Rep. 2018;8(1):254.

61 George JF, Agarwal A. Hydrogen: another gas with therapeutic potential. Kidney Int. 2010; 77(2):85-7.

62 Cardinal JS, Zhan J, Wang Y, Sugimoto R, Tsung A, McCurry KR, et al. Oral hydrogen water prevents chronic allograft nephropathy in rats. Kidney Int. 2010;77(2):101-9.

63 Nisula S, Kaukonen KM, Vaara ST, Korhonen AM, Poukkanen M, Karlsson S, et al. Incidence, risk factors and 90-day mortality of patients with acute kidney injury in Finnish intensive care units: the FINNAKI study. Intensive Care Med. 2013;39(3):420-8.

64 Skube SJ, Katz SA, Chipman JG, Tignanelli CJ. Acute kidney injury and sepsis. Surg Infect. 2018;19(2):216-24.
65 Zhang J, Ankawi G, Sun J, Digvijay K, Yin Y, Rosner $\mathrm{MH}$, et al. Gut-kidney crosstalk in septic acute kidney injury. Crit Care. 2018; 22(1):117.

66 Qiu R, Yao W, Ji H, Yuan D, Gao X, Sha W, et al. Dexmedetomidine restores septic renal function via promoting inflammation resolution in a rat sepsis model. Life Sci. 2018;204: $1-8$.

67 Yao W, Guo A, Han X, Wu S, Chen C, Luo C, et al. Aerosol inhalation of a hydrogen-rich solution restored septic renal function. Aging. 2019;11(24):12097-113.

68 Xin HG, Zhang BB, Wu ZQ, Hang XF, Xu WS, Ni W, et al. Consumption of hydrogenrich water alleviates renal injury in spontaneous hypertensive rats. Mol Cell Biochem. 2014;392(1-2):117-24.

69 Guan P, Sun ZM, Luo LF, Zhao YS, Yang SC, Yu FY, et al. Hydrogen gas alleviates chronic intermittent hypoxia-induced renal injury through reducing iron overload. Molecules. 2019;24(6):1184.
70 Shi Q, Liao KS, Zhao KL, Wang WX, Zuo T, Deng $\mathrm{WH}$, et al. Hydrogen-rich saline attenuates acute renal injury in sodium taurocholate-induced severe acute pancreatitis by inhibiting ROS and NF-kB pathway. Mediators Inflamm. 2015;2015:685043.

71 Homma K, Yoshida T, Yamashita M, Hayashida K, Hayashi M, Hori S. Inhalation of hydrogen gas is beneficial for preventing contrast-induced acute kidney injury in rats. Nephron Exp Nephrol. 2015. Epub ahead of print.

72 Saitoh Y, Harata Y, Mizuhashi F, Nakajima M, Miwa N. Biological safety of neutral-pH hydrogen-enriched electrolyzed water upon mutagenicity, genotoxicity and subchronic oral toxicity. Toxicol Ind Health. 2010;26(4): 203-16.

73 Nakao A, Toyoda Y, Sharma P, Evans M, Guthrie N. Effectiveness of hydrogen rich water on antioxidant status of subjects with potential metabolic syndrome-an open label pilot study. J Clin Biochem Nutr. 2010;46(2): 140-9. 